\title{
Stellar population in LLAGN
}

\author{
Rosa M. González Delgado ${ }^{1}$ \\ ${ }^{1}$ Instituto de Astrofísica de Andalucía (CSIC), Granada, Spain email:rosa@iaa.es
}

\begin{abstract}
LLAGN that include low-ionization nuclear emission-line regions (LINERs), and transition-type objects (TOs) represent the most common type of nuclear activity. Here, a study of the central stellar population of LLAGN is presented. Our goal is to search for spectroscopic signatures of young and intermediate age stars, and to investigate their relationship with the ionization mechanism in LLAGN. The method used is based on the stellar population synthesis of the UV-optical continuum of the innermost (20-100 pc) regions in these galaxies. Half of the LINERs and TOs of the Palomar catalogue are analysed. It is found that weak-[OI] $([\mathrm{OI}] / \mathrm{H} \alpha \leqslant$ 0.25) LLAGN have an intermediate age stellar population that dominates the optical light. But young stellar clusters dominate the UV continuum in these objects. These clusters can co-exist with a black-hole in spatial scales of a few pc. Most of the strong-[OI] LLAGN have a predominantly old stellar population. These results suggest that young and intermediate age stars do not play a significant role in the ionization of LLAGN with strong [OI].
\end{abstract}

\section{Introduction}

Low Luminosity Active Galactic Nuclei (LLAGN) constitute a sizeable fraction of the nearby AGN population. These include low-luminosity Seyferts, low-ionization nuclear emission-line regions (LINERs), and transition-type objects (TOs) whose properties are in between classical LINERs and HII nuclei. LLAGN comprise about $1 / 3$ of all bright galaxies $\left(\mathrm{B}_{T} \leqslant 12.5\right)$ and are the most common type of AGNs (Ho, Filippenko \& Sargent 1997a; hereafter HFS97). LLAGN could constitute a rather mixed phenomena as suggested by the several excitation mechanisms that have been proposed to explain the origin of their energy source. Among these mechanisms are shocks, photoionisation by a non-stellar UV/X-ray continuum (AGN), and photoionisation by hot stars (see e.g. review by Fillipenko 1996). These hot stars could be the product of the evolution of massive stars (Terlevich \& Melnick 1985; Barth \& Shields 2000) or of intermediate mass stars (the post-AGB stars investigated by Binette et al. 1994 and Taniguchi, Shioya, \& Murayama 2000). Further evidence in favor of the starburst scenario come from the detection of stellar wind lines in the ultraviolet spectra of a few weak-[OI] LINERs and TOs (Maoz et al 1998; Colina et al 2002).

The results obtained in several projects (e.g. González Delgado et al 2004; Cid Fernandes et al 2004; Colina et al 2002) which examine the central stellar population of these objects are summarized here, to find out the role of starbursts in LLAGN.

\section{Sample and Observations}

The sample of LLAGN comprises about half of the LINERs and TOs of the Palomar catalogue (HFS97). We divide the objects in two subtypes according with their $[\mathrm{OI}] / \mathrm{H} \alpha$ ratio. In total, we have 24 strong-[OI] $([\mathrm{OI}] / \mathrm{H} \alpha \geqslant 0.25)$ and 47 weak-[OI] LLAGN. The median morphological type (and distance) is $\mathrm{S} 0$ (17 Mpc) and Sab (23 Mpc) for weak and strong-[OI] LLAGN, respectively. We have obtained ground-based optical spectra (3400-5500 $\AA$ ) for 51 objects, using ALFOSC at the NOT and the $2.2 \mathrm{~m}$ telescope at 
KPNO. We have retrieved from the HST archive STIS (G430L) spectra for 28 LLAGN and WFPC2 optical images for all galaxies. In addition, we have analized UV (STIS or FOS) for a few of them.

\section{Optical Results}

The central stellar population is characterized by: (1) searching for the presence of the broad WR bump at the blue optical range (a blend of broad He II $\lambda 4686, \mathrm{~N}$ III $\lambda 4640$ and C IV $\lambda 4650$ ); (2) detecting the absorption lines of HeI and the high order HI Balmer lines (HOBLs); (3) examining the metallic lines, such as CaIIK, G band and MgII. The WR bump probes the presence of very young stars (few Myr old), while HeI and HOBLs probe the young (10-50 Myr) and intermediate (100-1000 Myr) age stars (González Delgado, Leitherer \& Heckman 1999), and the metallic lines probe the intermediate and old stellar population. We have followed several methods: a) empirical comparison with normal galaxies; b) performing stellar population synthesis of the optical continuum. We have also correlated the stellar properties with the emission line ratios which may distinguish between possible excitation sources for the gas.

Our main findings are: (1) No features due to Wolf-Rayet stars were convincingly detected in the STIS and ground-based spectra. (2) Young stars contribute very little to the optical continuum in the ground-based aperture. However, the fraction of light provided by these stars is higher than $10 \%$ in most of the weak-[OI] $([\mathrm{OI}] / \mathrm{H} \alpha \leqslant 0.25)$ LLAGN STIS spectra. (3) Intermediate age stars contribute significantly to the optical continuum of these nuclei (Figure 1 and 3). This population is more frequent in objects with weak than with strong [OI]. Weak-[OI] LLAGN that have young stars stand out for their intermediate age population. (4) Most of the strong-[OI] LLAGN have predominantly old stellar population. A few of these objects also show a featureless continuum that contributes significantly to the optical continuum (Figure 1 and 3). These results suggest that young and intermediate age stars do not play a significant role in the ionization of LLAGN with strong [OI]. However, the ionization in weak-[OI] LLAGN with young and/or intermediate age population could be due to stellar processes. A comparison of the properties of these objects with Seyfert 2 galaxies that harbor a nuclear starburst, suggests that weak-[OI] LLAGN are the lower luminosity counterparts of the Seyfert 2 composite nuclei.

\section{UV Results}

Many LLAGN show central knots at optical and/or UV wavelengths whose origin can not be known based only on their central morphology (see Figure 2). They can be super stellar clusters (SSCs) or a compact unresolved AGN. In fact, SSCs are a common phenomenon in spirals (Carollo et al 2002), and in galaxies with nuclear starbursts (Meurer et al 1995). UV observations of these knots can clarify their origin because these wavelengths are the most direct tracer of unobscured young stellar clusters. Massive stars emit most of their flux at these wavelengths and they show strong UV P-Cygni resonance lines formed in their winds. These lines can unambiguously discriminate between the stellar and non-stellar (AGN) origin of nuclear knots. Additionally, these lines can constrain also the properties of the stellar clusters, such as age and metallicity.

UV spectra of a few LLAGN have revealed the existence of a young stellar cluster at their nuclei (Maoz et al 1998). The best example is found in NGC 4303 (Colina et al 2002), whose UV image shows a ring of star forming clusters spiraling towards the nucleus, where there is a compact 3 pc radius core. The STIS UV spectrum of this core 

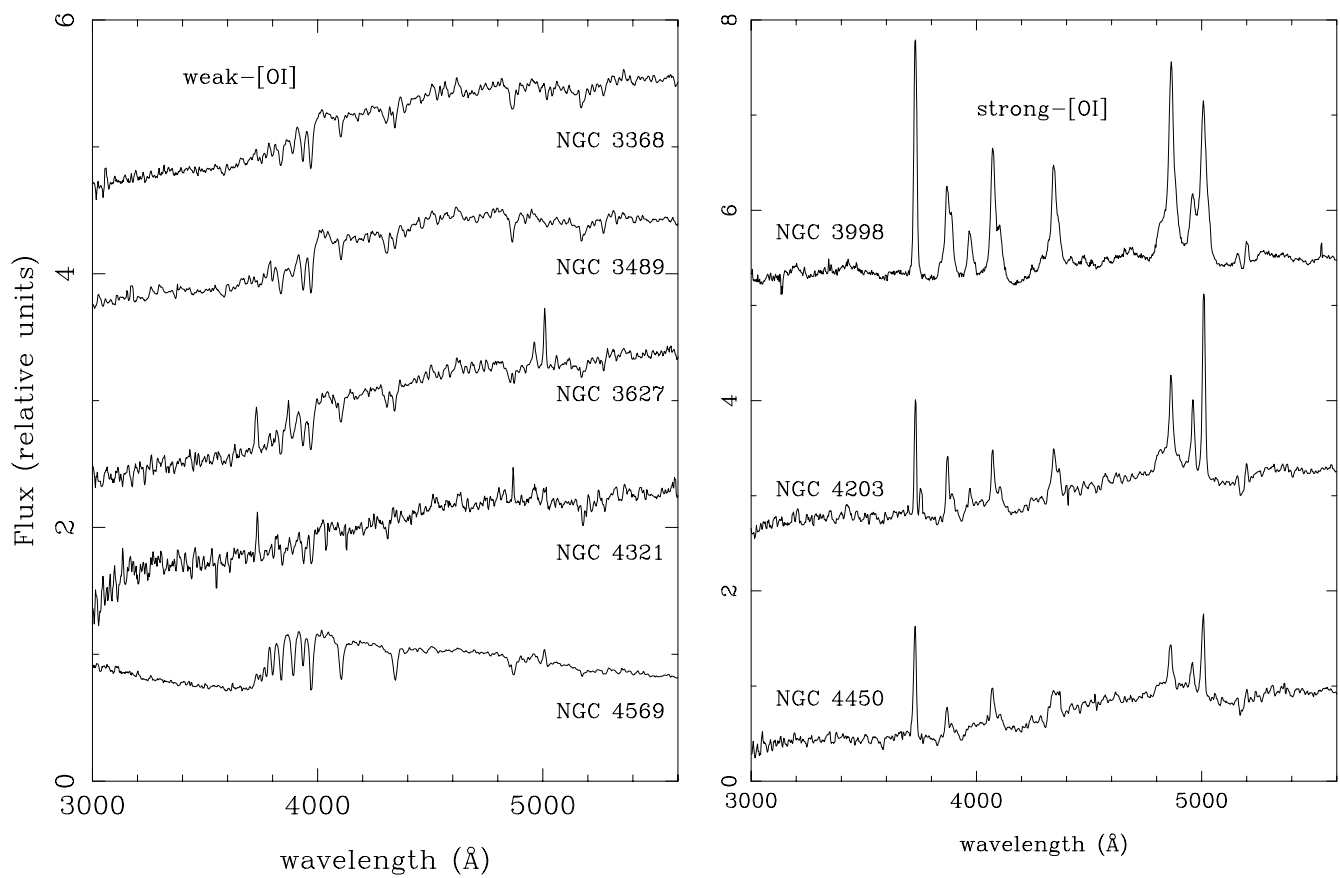

Figure 1. Nuclear spectra $\left(0.3 \times 0.2^{\prime \prime}\right)$ of LLAGN obtained with STIS+HST. Examples of weak-[OI] LLAGN with optical continuum dominated by intermediate age population are shown on the left, and strong-[OI] LLAGN with diluted stellar lines due to the AGN.

shows P-Cygni wind lines that prove that the core is a $4 \mathrm{Myr}$ old stellar cluster with a mass of $2-3 \times 10^{5} \mathrm{M} \odot$ at solar metallicity. Another good example is found in NGC 3507 (Figure 2). Even though these clusters dominate the UV emission, their contribution to longer wavelengths is low, the optical continuum being dominated by intermediate age stars. However, additional observations obtained by Chandra find that the central 1.5-5 keV emission can not be provided by the young stellar cluster, and a massive black hole may be responsible for the hard X-ray emission (Jiménez Bailón et al 2003). These studies show that the central structure of LLAGN is very complex because a young stellar cluster, with intermediate age stars can co-exist with a black hole in a spatial scale of a few pc. A multi-wavelengths analysis is needed to constrain the contributions of the AGN and the starburst to the central total luminosity.

\section{Acknowledgements}

I acknowledge support from the IAU, and the conference organizers, and I am grateful to my collaborators, Roberto Cid Fernandes, Luis Colina, Tim Heckman, Claus Leitherer, Lucimara Martins, Miguel Mas-Hesse, Enrique Pérez, Thaisa Storchi-Bergmann, and Henrique Schmitt, with whom I have done the work discussed here.

\section{References}

Barth, A., \& Shields, J. C. 2000, PASP, 112, 753

Binette, L., Magris, C. G., Stasinska, G., \& Bruzual, A. G. 1994, A\&A, 292, 13

Carollo, C. M., et al. 2002, ApJ, 123, 159

Cid Fernandes, R., et al. 2004, ApJ, 605, 105 


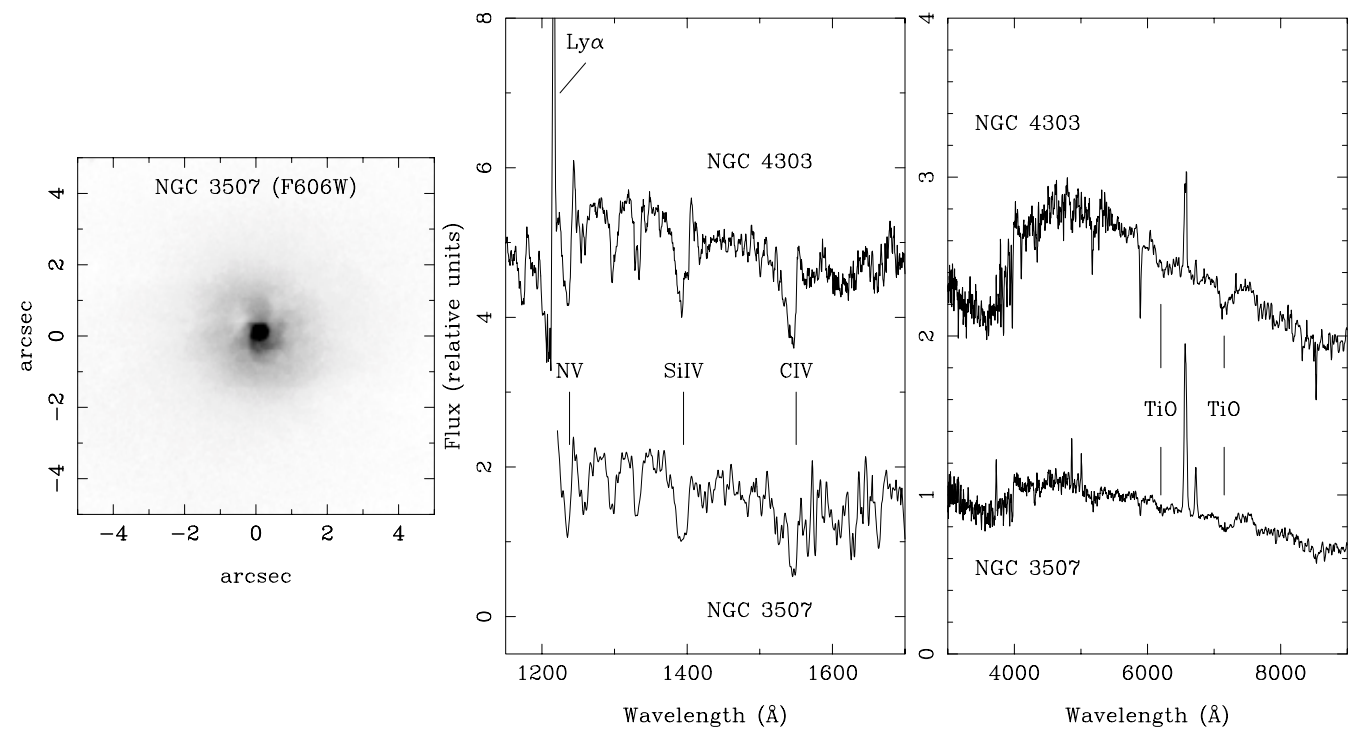

Figure 2. WFPC2 central structure of NGC 3507 and UV (left) and optical (right) spectra of the nuclear cluster in NGC 3507 and NGC 4303.

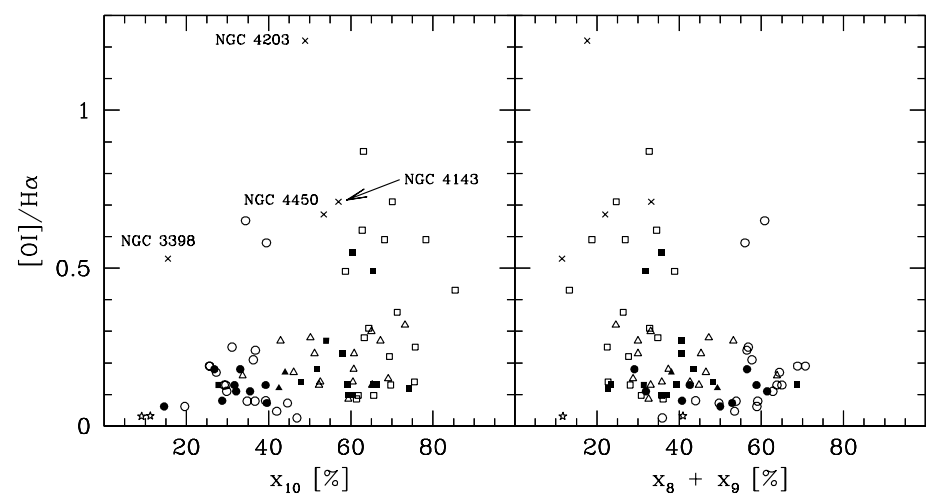

Figure 3. $[\mathrm{OI}] / \mathrm{H} \alpha$ vs fraction of the light provided by: (a) old (10Gyr), and (b) intermediate $(1 \mathrm{Gyr}+100 \mathrm{Myr})$ age population. Filled and open symbols are used for STIS and ground-based observations, respectively.

Colina, L., González Delgado, R. M., Mas-Hesse, J. M., \& Leitherer, C. 2002, ApJ, 579, 545

Ferrarese, L., \& Merrit, D. 2000, ApJ, 539, L9

Filippenko, A. V. 1996, ASP Conf. Series, 103, 17

González Delgado, R. M., et al. 2004, ApJ, 605, 127

Ho, L. C., Filippenko, A. V., \& Sargent, W. L. W. 1997, ApJS, 112, 315

Jiménez-Bailón, E., et al. 2003, ApJ, 593, 127

Maoz, D., et al. 1998, AJ, 116, 55

Meurer, G., et al. 1995, AJ, 110, 2665

Taniguchi, Y., Shioya, Y., \& Murayama, T. 2000, AJ, 120, 1265

Terlevich, R., \& Melnick, J. 1985, MNRAS, 213, 841 